\title{
Puerarin alleviates the ototoxicity of gentamicin by inhibiting the mitochondria-dependent apoptosis pathway
}

\author{
PING NIU ${ }^{1,2^{*}}$, YUXUAN SUN ${ }^{3 *}$, SHIYI WANG ${ }^{4},{\text { GUANG } \mathrm{LI}^{4}, \text { XIAOMIN TANG }}^{3}$, \\ JIAQIANG SUN ${ }^{3}$, CHUNCHEN PAN ${ }^{3}$ and JINGWU SUN ${ }^{3}$ \\ ${ }^{1}$ Department of Otolaryngology-Head and Neck Surgery, Anhui Provincial Hospital, Cheeloo College of Medicine, \\ Shandong University, Jinan, Shandong 250012; ${ }^{2}$ Department of Otolaryngology, Qingzhou Hospital Affiliated to \\ Shandong First Medical University, Qingzhou People's Hospital, Qingzhou, Shandong 262500; \\ ${ }^{3}$ Department of Otolaryngology-Head and Neck Surgery, Anhui Provincial Hospital, \\ Hefei, Anhui 230001; ${ }^{4}$ Department of Otolaryngology-Head and Neck Surgery, \\ Affiliated Hospital of Yangzhou University, Yangzhou, Jiangsu 225001, P.R. China
}

Received February 28, 2021; Accepted September 22, 2021

DOI: $10.3892 / \mathrm{mmr} .2021 .12491$

\begin{abstract}
Gentamicin (GM) is a commonly used antibiotic, and ototoxicity is one of its side effects. Puerarin (PU) is an isoflavone in kudzu roots that exerts a number of pharmacological effects, including antioxidative and free radical scavenging effects. The present study investigated whether PU could protect against GM-induced ototoxicity in C57BL/6J mice and House Ear Institute-Organ of Corti 1 (HEI-OC1) cells. C57BL/6J mice and HEI-OC1 cells were used to establish models of GM-induced ototoxicity in this study. Auditory brainstem responses were measured to assess hearing thresholds, and microscopy was used to observe the morphology of cochlear hair cells after fluorescent staining. Cell viability was examined with Cell Counting Kit-8 assays. To evaluate cell apoptosis and reactive oxygen species (ROS) production, TUNEL assays, reverse transcription-quantitative PCR, DCFH-DA staining, JC-1 staining and western blotting were performed. PU protected against GM-induced hearing damage in $\mathrm{C} 57 \mathrm{BL} / 6 \mathrm{~J}$ mice. PU ameliorated the morphological changes of mouse cochlear hair cells and reduced the apoptosis rate of HEI-OC1 cells after GM-mediated damage. GM-induced ototoxicity may be closely related to the upregulation of p53 expression and the activation of endogenous mitochondrial
\end{abstract}

Correspondence to: Dr Chunchen Pan or Professor Jingwu Sun, Department of Otolaryngology-Head and Neck Surgery, Anhui Provincial Hospital, 17 Lujiang Road, Hefei, Anhui 230001, P.R. China

E-mail: panchunchen@hotmail.com

E-mail: entsun@ustc.edu.cn

*Contributed equally

Key words: puerarin, gentamicin, ototoxicity, apoptosis, reactive oxygen species, p53 apoptosis pathways, and PU could protect cochlear hair cells from GM-mediated damage by reducing the production of ROS and inhibiting the mitochondria-dependent apoptosis pathway.

\section{Introduction}

Gentamicin (GM) is an aminoglycoside antibiotic that is commonly used in the clinic (1). For more than half a century, despite the advantages of aminoglycosides in terms of their stable properties, broad antibacterial spectrum, strong bactericidal power and low cost, a large number of clinical reports and animal experiments have also proven that aminoglycosides induce ototoxicity (2), which severely limits their clinical application. Current studies have found that the mechanism underlying aminoglycoside ototoxicity may be that aminoglycoside antibiotics can accumulate in cochlear hair cells, and mitochondria are the main site of accumulation $(3,4)$. Moreover, the hair cells located in the basal turn of the basilar membrane have a stronger absorption capacity than those located in the apical turn (5). The damage to cochlear hair cells caused by aminoglycosides is associated with the activity of oxygen radicals, and the expression of caspase- 3 is also subsequently increased (6). Thus, GM-induced ototoxicity may be closely related to the overproduction of reactive oxygen species (ROS) in cochlear hair cells and the activation of endogenous mitochondrial apoptosis pathways.

Puerarin (PU) is the main active ingredient among the isoflavones of wild Pueraria and dried Pueraria (7). Isoflavones are aromatic oxyheterocyclic compounds and effective antioxidants that prevent the formation of oxygen free radicals and exert biological antioxidant effects (8). Based on a number of animal experiments and clinical studies, PU has antioxidant, anti-ageing, anti-inflammatory and anti-osteoporosis activities and is also used to treat hangovers and hypoglycaemia $(9,10)$; in addition, PU is mainly used in the treatment of neurological diseases and cardiovascular diseases $(11,12)$. The mechanism underlying the various pharmacological effects of PU has 
been thoroughly studied and has attracted increasing attention from researchers. PU can improve the learning and memory abilities of ageing mice, and the mechanism underlying its anti-ageing effects is related to the inhibition of mitochondrial dysfunction and the enzyme activity of caspase-3 (13). PU can also significantly improve the activity of superoxide dismutase in ageing mice and enhance its ROS scavenging function. PU may inhibit the expression of caspase- 3 and Bax, which are closely related to cell apoptosis, and promote the protein expression of Bcl-2 (11,12). In addition, it has been reported that PU is useful in treating alcoholic liver disease as an antidote and anti-drinking agent (14). However, the effects of PU on GM-induced ototoxicity have not yet been reported.

Apoptosis is a pathophysiological process in which various proteins or factors are involved. p53 is considered to be an important gene that mediates cell apoptosis and plays an important regulatory role in various cellular stress responses (15). The mechanism by which p53 functions is extremely complex, and p53 plays vital roles in the signalling pathways mediated by endogenous regulatory factors. When cells are stimulated by apoptotic signals, the expression of p53 is increased; p53 regulates the expression of Bcl-2 family proteins through transcription-dependent pathways, and p53 can also directly act on mitochondria-mediated apoptosis pathways. In fact, $\mathrm{H}_{2} \mathrm{O}_{2}$, the most important ROS in redox signalling, has been shown to trigger a typical DNA damage response pathway and the subsequent activation of p53 and apoptosis $(16,17)$. GM-induced ototoxicity may be closely related to the overproduction of ROS in cochlear hair cells, the upregulation of 553 protein expression and the activation of endogenous mitochondrial apoptosis pathways. Thus, in the present study it was hypothesized that PU may protect cochlear hair cells from GM-mediated damage by reducing ROS production and inhibiting the mitochondria-dependent apoptosis pathway.

\section{Materials and methods}

Animal experiments and drugs. The animal protocols followed the guidelines of the Institutional Animal Care and Use Committee of University of Science and Technology of China (Jinan, China), and the experiments were conducted in accordance with the National Institutes of Health (NIH) Guide for the Care and Use of Animals in Laboratory Experiments (18). The study was approved by the Institutional Animal Care and Use Committee of University of Science and Technology of China. A total of 24 male C57BL/6J mice ( 8 days old; $4.5 \pm 0.3 \mathrm{~g}$ ) were purchased from Changzhou Cavens Experimental Animal Co., Ltd. The mice were housed in an SPF facility, where they were under a controlled temperature of $25 \pm 2{ }^{\circ} \mathrm{C}$, a humidity of $55 \pm 5 \%$ and a 12-h day/night cycle, and where the mice were allowed to eat and drink ad libitum. The health and behaviour of the mice were checked every day. Animals were maintained in accordance with the Guidelines for the Care and Use of Laboratory Animals (18). When the mice were 6 weeks old, the 24 mice were divided into four groups (six mice in each group): The control group; PU group; GM group; and $\mathrm{GM}+\mathrm{PU}$ group. The mice in the control group were intraperitoneally injected with $1 \mathrm{ml}$ physiological saline once a day for 10 days. The mice in the PU group were intraperitoneally injected with 100 mg/kg PU (Sigma-Aldrich; Merck KGaA) once a day for 10 days (19). The mice in the GM group were first intraperitoneally injected with $1 \mathrm{ml}$ physiological saline once a day for 3 consecutive days and then intraperitoneally injected with $200 \mathrm{mg} / \mathrm{kg}$ GM (Sigma-Aldrich; Merck KGaA) once a day for 7 days (20). The mice in the GM + PU group were first intraperitoneally injected with $100 \mathrm{mg} / \mathrm{kg} \mathrm{PU}$ once a day for 3 consecutive days and then intraperitoneally injected with $100 \mathrm{mg} / \mathrm{kg}$ PU and $200 \mathrm{mg} / \mathrm{kg} \mathrm{GM}$ once a day for 7 consecutive days. In the entire study, no mice died accidentally. All efforts were made to minimize suffering. All the 24 mice were anaesthetized with an intraperitoneal injection of a combination of ketamine $(100 \mathrm{mg} / \mathrm{kg})$ and xylazine $(15 \mathrm{mg} / \mathrm{kg})$ (21) At the end of the experiments, the mice were administrated with $30 \%$ volume displacement rate of $\mathrm{CO}_{2}$ for euthanasia. Animal death was confirmed by observing cardiac arrest and mydriasis.

Auditory brainstem response $(A B R)$ test. The ABR thresholds of the four groups were measured in the 8th week. The mice were anaesthetized by intraperitoneal injection with a combination of ketamine $(100 \mathrm{mg} / \mathrm{kg})$ and xylazine $(15 \mathrm{mg} / \mathrm{kg})(21)$, and the mice were kept warm during the ABR recording. ABR measurements were performed by using Tucker-Davis Technology System hardware and software (Tucker-Davis Technologies). The recording electrode was subcutaneously inserted into the tissue at the vertex, and the reference and ground electrodes were subcutaneously placed behind the ears. The ABR was measured with broadband clicks and pure tones at frequencies of $8,12,16,24$ and $32 \mathrm{kHz}$ with 1,024 stimulus repetitions per record, and the stimulus sound was decreased from $90 \mathrm{~dB}$ SPL to $10 \mathrm{~dB}$ SPL successively at 10-dB SPL intervals. When the electrophysiological response to the stimulus sound disappeared, the lowest stimulus sound that triggered a response was defined as the auditory threshold of the mouse tested at this frequency.

Treatment of cochlear basal membranes and staining. After the ABR was detected, the mice were anaesthetized, and perfusion was performed with normal saline and $4 \%$ paraformaldehyde. The head was removed, the temporal bone was removed and the cochlea was separated. The cochlea was immersed in a $10 \%$ EDTA solution and stored at $4^{\circ} \mathrm{C}$ for 7 days. The basilar membrane was then isolated from the cochlea under an anatomical microscope. The basilar membrane was fixed in $10 \%$ formaldehyde solution at room temperature for $15 \mathrm{~min}$ and fluorescence staining was performed with phalloidin $(1: 100$, red $)$ at room temperature in the dark for $30 \mathrm{~min}$. Fluorescence microscopy was used for observation and imaging. The number of outer and inner hair cells in each segment of the basilar membrane was quantified per $100 \mu \mathrm{m}$. Images were captured using a fluorescence microscope (Leica Corporation).

Cell culture and treatment. House Ear Institute-Organ of Corti 1 (HEI-OC1) cells were derived from a murine Corti organ; this cell line is a type of auditory cell line and has properties similar to those of hair cells. HEI-OC1 cells were donated by Professor Renjie Chai of Southeast University. HEI-OC1 cells were cultured in high-glucose medium (Gibco; Thermo Fisher Scientific, Inc.) containing $10 \%$ foetal bovine 
serum (Gibco; Thermo Fisher Scientific, Inc.) in a $10 \% \mathrm{CO}_{2}$ incubator at $33^{\circ} \mathrm{C}$. The HEI-OC1 cells in the control group were treated with no drugs. The cells in the GM, PU and $\mathrm{H}_{2} \mathrm{O}_{2}$ groups were treated with $1.0 \mathrm{mM} \mathrm{GM}, 100 \mu \mathrm{g} / \mathrm{ml} \mathrm{PU}$ and $0.1 \mathrm{mM} \mathrm{H}_{2} \mathrm{O}_{2}$ for $24 \mathrm{~h}$, respectively. The cells in the $\mathrm{GM}+\mathrm{PU}$ group were pre-treated with $100 \mu \mathrm{g} / \mathrm{ml} \mathrm{PU}$ for $2 \mathrm{~h}$, followed by a combination treatment with $1.0 \mathrm{mM} \mathrm{GM}$ for $24 \mathrm{~h}$. The cells in the $\mathrm{H}_{2} \mathrm{O}_{2}+\mathrm{PU}$ group were pre-treated with $100 \mu \mathrm{g} / \mathrm{ml} \mathrm{PU}$ for $2 \mathrm{~h}$, followed by a combination treatment with $0.1 \mathrm{mM} \mathrm{H}_{2} \mathrm{O}_{2}$ for $24 \mathrm{~h}$. All treatments were at $33^{\circ} \mathrm{C}$.

Cell viability. HEI-OC1 cells were seeded in a 96-well plate $\left(5,000\right.$ cells per well) and incubated overnight at $33^{\circ} \mathrm{C}$ in $10 \% \mathrm{CO}_{2}$. The cells were treated with different concentrations of GM $(0.1,0.2,0.5,1.0$ and $2.0 \mathrm{mM})$ and PU $(5,10,20$, $50,100,200$ and $300 \mu \mathrm{g} / \mathrm{ml}$ ) for $24 \mathrm{~h}$. A total of $10 \mu \mathrm{l} \mathrm{Cell}$ Counting Kit-8 (CCK-8; Sangon Biotech Co., Ltd.) was added to the treated cells in each well and incubated at $37^{\circ} \mathrm{C}$ for $1 \mathrm{~h}$. The absorbance at $450 \mathrm{~nm}$ was determined with an ELISA reader (Multiskan MK3). The cell viability was calculated as (\%) (OD assay - OD blank) / (OD control - OD blank) x100\%.

Flow cytometry analysis of cell apoptosis. The treated cells were collected and transferred into a centrifuge tube. After centrifugation $\left(1,000 \mathrm{x} \mathrm{g}, 4^{\circ} \mathrm{C}, 5 \mathrm{~min}\right)$, the cells were resuspended in $100 \mu \mathrm{l}$ binding buffer. An Annexin V-APC/7-AAD apoptosis kit (BD Biosciences) was used for double staining in the dark. The apoptosis rate of the HEI-OC1 cells was measured by flow cytometry (CytoFLEX S; Beckman Coulter, Inc.). CytExpert (v2.0; Beckman Coulter, Inc.) was used to analyze the data.

Detection of ROS. The intracellular ROS levels were detected by DCFH-DA staining (cat. no. E004; Nanjing Jiancheng Bioengineering Institute). DCFH-DA $(10 \mu \mathrm{M})$ was added to the treated cells in a 6 -well plate, at $1 \times 10^{6}$ cells per well, and incubated at $33^{\circ} \mathrm{C}$ in $10 \% \mathrm{CO}_{2}$ for $2 \mathrm{~h}$. The cells were observed and photographed under a fluorescence microscope. Then, ROS production of the HEI-OC1 cells was measured by flow cytometry (Beckman Coulter, Inc.) (22). CytExpert (v2.0; Beckman Coulter, Inc.) was used to analyze the data.

TUNEL assay. A TUNEL assay was used to investigate the morphological characteristics of apoptosis. The treated cells were fixed with $4 \%$ paraformaldehyde for $30 \mathrm{~min}$ at $20^{\circ} \mathrm{C}$. After 20 min of treatment with $0.1 \%$ Triton X-100, TUNEL working solution (Beyotime Institute of Biotechnology) was added to the cells for $1 \mathrm{~h}$ at $37^{\circ} \mathrm{C}$. DAPI staining was performed for $2 \mathrm{~min}$ at $20^{\circ} \mathrm{C}$. The treated HEI-OC1 cells were then photographed and analyzed under a fluorescence microscope. Images were captured using a fluorescence microscope (Leica Corporation). For each sample, $>5$ areas were randomly selected for analysis.

Mitochondrial fluorescent probe staining analysis. A JC-1 probe was used to measure mitochondrial depolarization in HEI-OC1 cells. Briefly, cells were treated in 6-well plates, at $1 \times 10^{6}$ cells per well, as indicated, and then the cells were incubated with an equal volume of JC-1 staining solution $(5 \mathrm{pg} / \mathrm{ml}$; Beyotime Institute of Biotechnology) at $37^{\circ} \mathrm{C}$ for $20 \mathrm{~min}$ and
Table I. Primers used in this study.

\begin{tabular}{ll}
\hline Gene & \multicolumn{1}{c}{ Sequences (5'-3') } \\
\hline GAPDH & F: GTATGACTCCACTCACGG \\
& R: GGTCTGGCTCCTGGAAGA \\
Bcl-2 & F: ATCGCCCTGTGGATGACTGAGT \\
& R: GCCAGGAGAAATCAAACAGAGGC \\
Bax & F: TCAGGATGCGTCCACCAAGAAG \\
& R: TGTGTCCACGGCGGCAATCATC \\
p53 & F: TCCGAAGACTGGATGACTGC \\
& R: GATCGTCCATGCAGTGAGGT \\
Caspase-3 & F: GGAAGCGAATCAATGGACTCTGG \\
& R: GCATCGACATCTGTACCAGACC
\end{tabular}

F, forward; R, reverse.

washed twice with PBS. The mitochondrial membrane potentials were monitored by determining the relative amounts of the emissions of mitochondrial JC-1 monomers or aggregates using fluorescence microscopy and flow cytometry. The excitation and emission wavelengths of 514 and $529 \mathrm{~nm}$, respectively, were used to detect the monomeric form of JC-1. JC-1 aggregation was detected at 585 and $590 \mathrm{~nm}$ (23). CytExpert (v2.0; Beckman Coulter, Inc.) was used to analyse the data.

$R N A$ extraction and reverse transcription-quantitative $P C R$ $(R T-q P C R)$. RT-qPCR was performed to detect the mRNA expression levels of Bax, caspase-3 and Bcl-2 in the HEI-OC1 cells. Total RNA was isolated from HEI-OC1 cells using RNAiso Plus ${ }^{\circledR}$ reagent (Takara Biotechnology Co., Ltd.). Next, according to the product specification, ReverTra-Plus ${ }^{\mathrm{TM}}$ (Toyobo Life Science) was used to reverse transcribe the RNA into cDNA. qPCR was performed using used TB Green ${ }^{\circledR}$ Premix Ex Taq ${ }^{\mathrm{TM}}$ II (Takara Biotechnology Co., Ltd.). The following thermocycling conditions were used for the qPCR: Initial denaturation at $95^{\circ} \mathrm{C}$ for $30 \mathrm{sec}$; followed by 40 cycles at $95^{\circ} \mathrm{C}$ for $5 \mathrm{sec}$ and $60^{\circ} \mathrm{C}$ for $34 \mathrm{sec}$. The primers used in this experiment (24) are shown in Table I. The mRNA expression levels were normalized to GAPDH expression, and gene expression was calculated by using the $2^{-\Delta \Delta \mathrm{Cq}}$ method in this study (25).

Western blotting. HEI-OC1 cells were lysed in RIPA buffer (Wuhan Servicebio Technology Co., Ltd.) or Cell Mitochondria Isolation kit (Beyotime Institute of Biotechnology). The total protein content was determined using a BCA Protein assay kit (Beyotime Institute of Biotechnology). Protein samples $(20 \mu \mathrm{g})$ were resolved by $10 \%$ SDS-PAGE (Wuhan Servicebio Technology Co., Ltd.), and subsequently transferred to polyvinylidene fluoride membranes (MilliporeSigma). The membranes were blocked with 5\% skimmed milk (P0216; Beyotime Institute of Biotechnology) for $1 \mathrm{~h}$ at room temperature. Then, the proteins were incubated with primary antibodies overnight in a $4^{\circ} \mathrm{C}$ refrigerator, followed by incubation with secondary antibodies [HRP-conjugated Affinipure Goat Anti-Rabbit IgG(H+L); cat. no. SA00001-2; 1:5,000, 


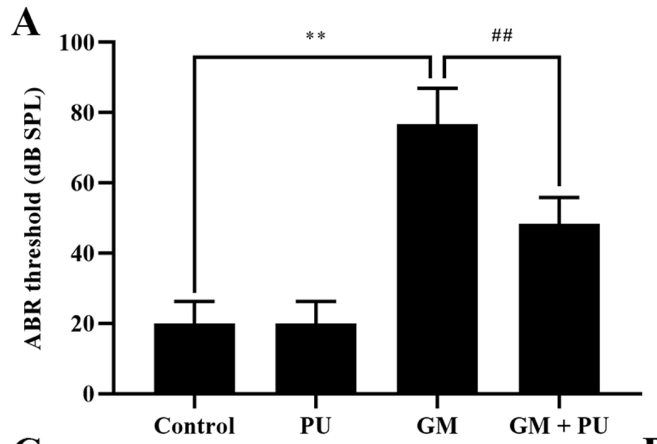

B
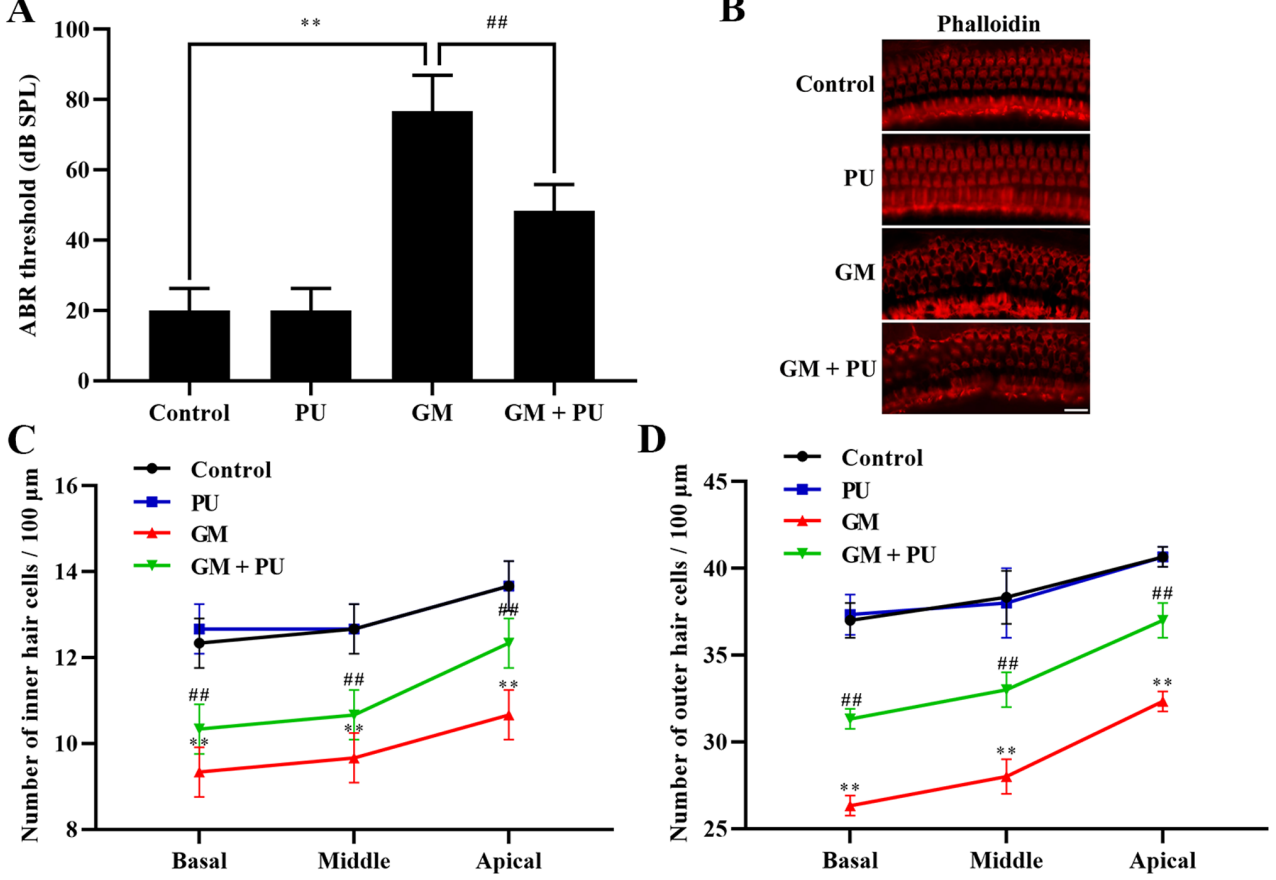

Figure 1. Effects of PU and GM in C57BL/6J mice. (A) Statistical analysis of ABR threshold. $\mathrm{n}=6 .{ }^{* * *} \mathrm{P}<0.01 \mathrm{GM}$ group vs. control group; ${ }^{* \#} \mathrm{P}<0.01 \mathrm{GM}+\mathrm{PU}$ group vs. GM group. (B) Image of basilar membrane stained with phalloidin. Scale bar, $20 \mu \mathrm{m}$. (C and D) Statistical analysis of the surviving outer and inner hair cells in the four groups. $\mathrm{n}=6 .{ }^{* *} \mathrm{P}<0.01 \mathrm{GM}$ group vs. control group; ${ }^{* \#} \mathrm{P}<0.01 \mathrm{GM}+\mathrm{PU}$ group vs. GM group. PU, puerarin; GM, gentamicin; ABR, auditory brainstem response.

ProteinTech Group, Inc.] at room temperature for $1 \mathrm{~h}$. An ECL kit (Wuhan Servicebio Technology Co., Ltd.) was used to detect the immunoreactive bands. Protein expression was detected using a Clinx ChemiScope 3300 (Clinx, Inc.). ImageJ software (v1.8; National Institutes of Health, Inc.) was used to quantify protein expression levels. GAPDH (cat. no. 5174; 1:1,000; Cell Signaling Technology, Inc.) and Cox IV (cat. no. 4850; 1:1,000; Cell Signaling Technology, Inc.) (26) were used as the loading controls. The primary antibodies used were specific for p53 (cat. no. 2524; 1:1,000; Cell Signaling Technology, Inc.), Bcl-2 (cat. no. sc-7382; 1:1,000; Santa Cruz Biotechnology, Inc.), cleaved-caspase-3 (cat. no. 9661; 1:1,000; Cell Signaling Technology, Inc.), caspase-3 (cat. no. 14220; 1:1,000; Cell Signaling Technology, Inc.), Bax (cat. no. 2772; 1:1,000; Cell Signaling Technology, Inc.) and cytochrome $c$ (Cyto C; cat. no. 11940; 1:1,000; Cell Signaling Technology, Inc.).

Statistical analysis. The statistical software GraphPad Prism 7 (GraphPad Software, Inc.) was used to analyse the significance of the data. All experimental results were independently repeated at least three times. All the data were collected from six or more samples in each of the experimental groups. The data are expressed as the mean \pm standard error of the mean, and one-way ANOVA followed by Tukey's post hoc test was performed. $\mathrm{P}<0.05$ was considered to indicate a statistically significant difference.

\section{Results}

PU protects against GM-induced hearing loss in C57BL/6J mice. The ABR threshold recording results showed that there was no difference between the PU and control groups. The threshold value of the GM group was significantly increased, with an increasing trend within the frequency range of 4 to $32 \mathrm{kHz}$, and the increase was more obvious at high frequencies (Fig. 1A). The threshold value of the GM + PU group was lower than that of the GM group.

PU attenuates GM-induced cochlear hair cell damage. The basal membrane of the cochlea and hair cells were stained with phalloidin (Fig. 1B). The results showed that the hair cells in the Control and PU groups were closely and neatly arranged. In the GM group, the hair cells were disorganized and partially absent, and the cilia were prostrate. Furthermore, the damage to hair cells in the basal and middle turns was more serious than that in the apical turn (Fig. 1C and D). The degree of hair cell damage in the GM + PU group was lower than that in the GM group.

PU improves the survival rate of HEI-OCl cells treated with GM. CCK-8 assays were used to detect the viability of HEI-OC1 cells (Fig. 2). The results showed that the viability of the HEI-OC1 cells treated with $1 \mathrm{mM} \mathrm{GM}$ was $\sim 50 \%$ (Fig. 2A). The HEI-OC1 cell viability was not affected by treatment with $5,10,20,50$ or $100 \mu \mathrm{g} / \mathrm{ml} \mathrm{PU}$, while 200 and $300 \mu \mathrm{g} / \mathrm{ml}$ PU decreased the viability of the HEI-OC1 cells (Fig. 2B). HEI-OC1 cells were pre-treated with different concentrations of PU $(10,50,100$ and $200 \mu \mathrm{g} / \mathrm{ml})$ for $2 \mathrm{~h}$, followed by a combination treatment with $1.0 \mathrm{~mm} \mathrm{GM}$ for $24 \mathrm{~h}$. It was demonstrated that the viability of the cells treated with $100 \mu \mathrm{g} / \mathrm{ml} \mathrm{PU}$ was the highest (Fig. 2C). Based on these results, treatment with $1 \mathrm{mM}$ $\mathrm{GM}$ for $24 \mathrm{~h}$ was considered to be the appropriate condition for the HEI-OC1 cell damage model, and $100 \mu \mathrm{g} / \mathrm{ml}$ PU was the optimal concentration for protecting HEI-OC1 cells from 

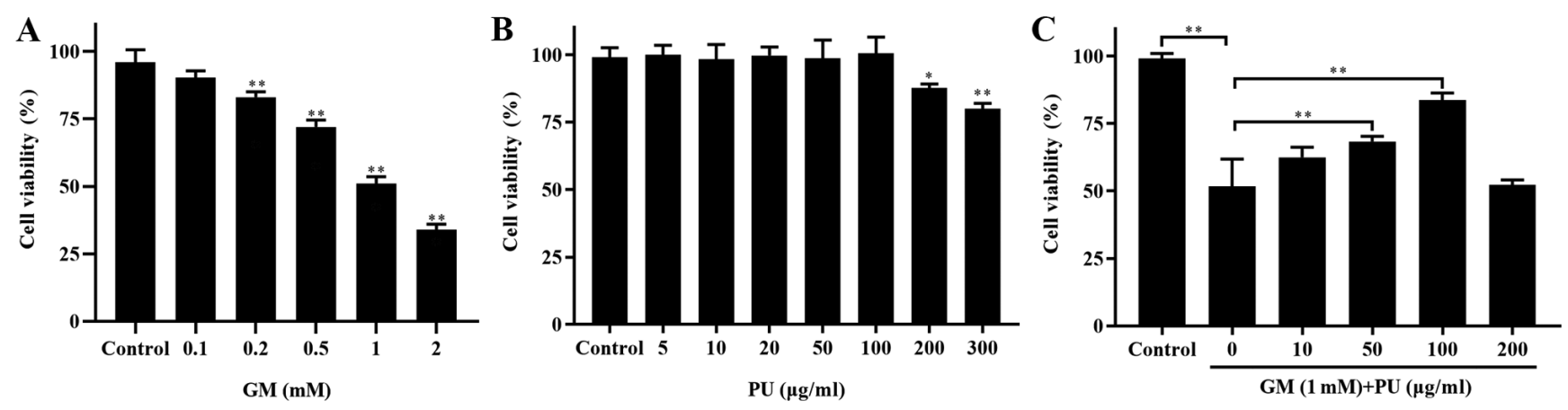

Figure 2. Effects of PU and GM in HEI-OC1 cells as determined via Cell Counting Kit-8 assays. (A) Viability of HEI-OC1 cells treated with different concentrations of GM for $24 \mathrm{~h} . \mathrm{n}=6{ }^{*}{ }^{* *} \mathrm{P}<0.01$. (B) Effect of PU on HEI-OC1 cell viability. Cells were treated with various concentrations of PU for $24 \mathrm{~h}$. $\mathrm{n}=6$. ${ }^{*} \mathrm{P}<0.05,{ }^{* *} \mathrm{P}<0.01$. (C) Cells were pre-treated with various doses of $\mathrm{PU}$ for $2 \mathrm{~h}$ prior to the addition of $1 \mathrm{mM}$ gentamicin and further cultured for $24 \mathrm{~h}$. $\mathrm{n}=6$. ${ }^{* *} \mathrm{P}<0.01$. PU, puerarin; GM, gentamicin; HEI-OC1, House Ear Institute-Organ of Corti 1.
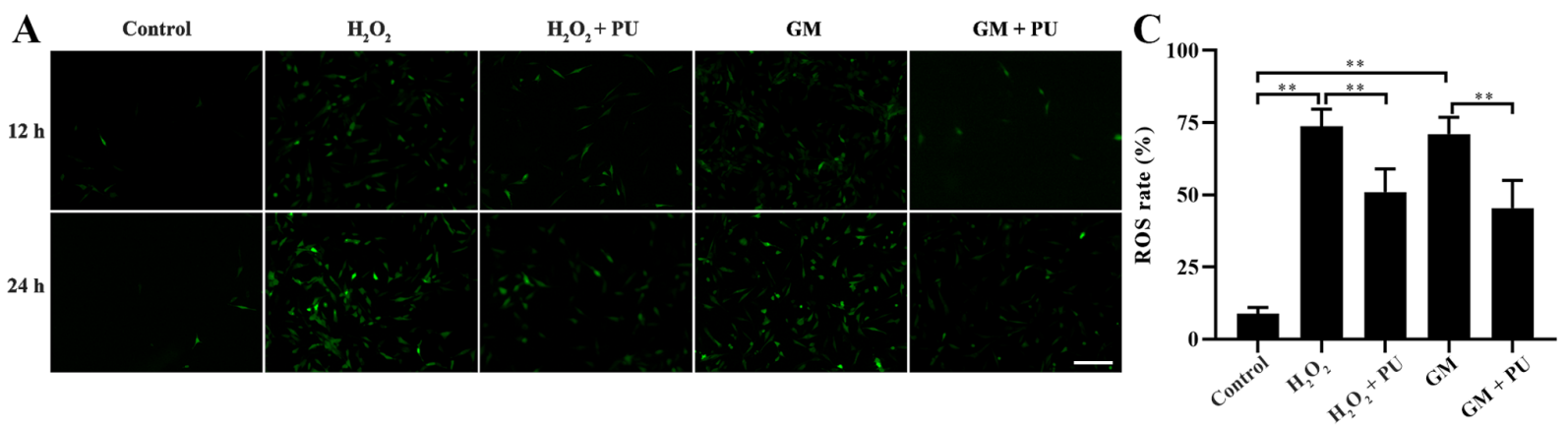

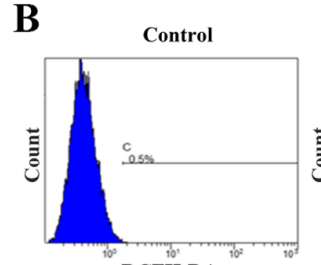

DCFH-DA

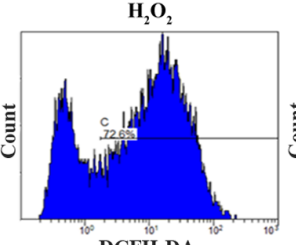

DCFH-DA

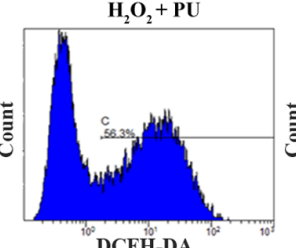

DCFH-DA

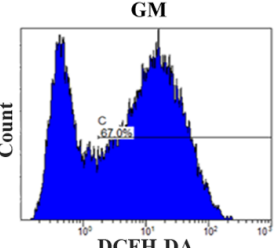

DCFH-DA

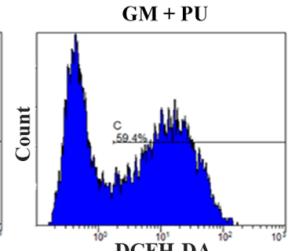

DCFH-DA

Figure 3. Effects of PU and GM on ROS in HEI-OC1 cells as determined by staining with DCFH-DA. (A) Image of HEI-OC1 cells stained with DCFH-DA (green). The treated cells were observed under a fluorescence microscope. Scale bar, $100 \mu \mathrm{m}$. (B) The fluorescence of DCFH-DA was detected to determine the levels of ROS in the cells by flow cytometry. (C) The ratios of ROS-positive cells and ROS-negative cells were statistically analysed. $\mathrm{n}=6$. ${ }^{* *} \mathrm{P}<0.01$. PU, puerarin; GM, gentamicin; HEI-OC1, House Ear Institute-Organ of Corti 1; ROS, reactive oxygen species.

GM-mediated damage. Therefore, the aforementioned treatment conditions were selected for subsequent cell experiments.

PU attenuates GM-induced oxidative stress in HEI-OCI cells. The cells in the Control, $\mathrm{H}_{2} \mathrm{O}_{2}, \mathrm{H}_{2} \mathrm{O}_{2}+\mathrm{PU}, \mathrm{GM}$ and $\mathrm{GM}+\mathrm{PU}$ groups were stained with fluorescent DCFH-DA for 12 and $24 \mathrm{~h}$, according to the manufacturer's instructions, and the cells were photographed under a fluorescence microscope (Fig. 3A). ROS were detected by DCFH-DA, and the intensity of green fluorescence was proportional to the level of ROS in the cells. The results indicated that the amount of intracellular ROS in the $\mathrm{H}_{2} \mathrm{O}_{2}$ and GM groups were notably increased in the $24 \mathrm{~h}$ staining group compared with the $12 \mathrm{~h}$ staining group, and PU could reduce the amount of intracellular ROS production induced by $\mathrm{H}_{2} \mathrm{O}_{2}$ and GM. Flow cytometry was used to detect the green fluorescence intensity of DCFH-DA in the cells after $24 \mathrm{~h}$ of treatment in each group (Fig. 3B). The statistical results also showed that $\mathrm{PU}$ could significantly reduce the ROS levels in the HEI-OC1 cells after treatment with $\mathrm{H}_{2} \mathrm{O}_{2}$ and GM (Fig. 3C).
PU attenuates GM-induced damage to mitochondrial membrane in HEI-OCl cells. JC-1 could aggregate in normal mitochondria and emit red fluorescence. The exposure of HEI-OC1 cells to GM $(1 \mathrm{mM})$ for $24 \mathrm{~h}$ resulted in the dissipation of the $\Delta \Psi \mathrm{m}$, which was shown by increased green fluorescence and decreased red fluorescence after JC-1 staining. Pre-treatment with PU $(100 \mu \mathrm{M})$ moderated this dissipation, indicating the protective effect of PU (Fig. 4A). The mitochondrial membrane potential of each group was evaluated with JC-1 stain by flow cytometry. Cells in the GM group displayed a lower ratio of mitochondria with normal potential. Pre-treatment of the cells with PU displayed a higher ratio (Fig. 4B and C). The western blotting results suggested that the Cyto $\mathrm{C}$ expression levels were significantly reduced in the mitochondria in the GM group compared with the Control group, but increased in the cytoplasm in the GM group. These changes were reversed following pre-treatment with PU (Fig. 4D and E).

PU inhibits the GM-induced apoptosis of HEI-OCl cells. The apoptosis of HEI-OC1 cells in each group was detected via 
A

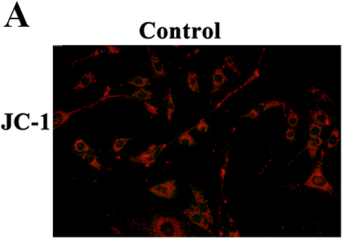

B
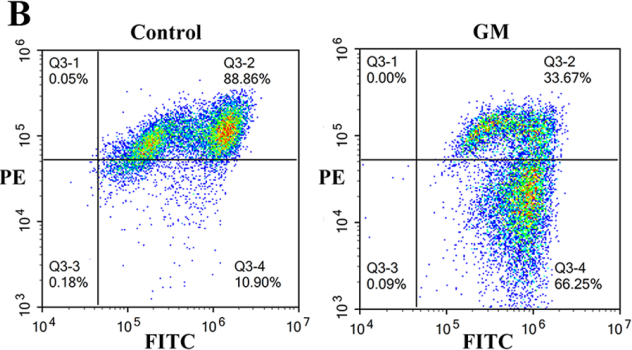

D

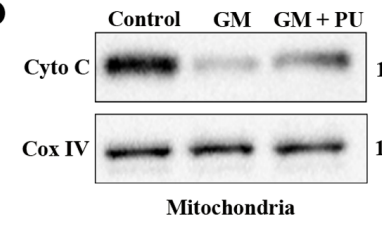

GM
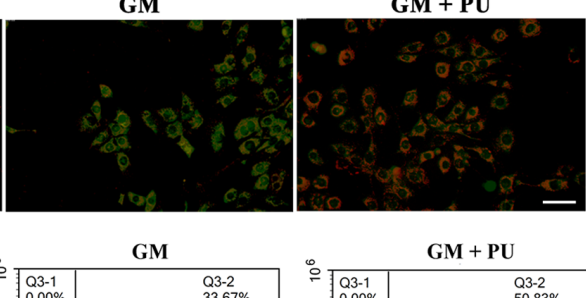

C

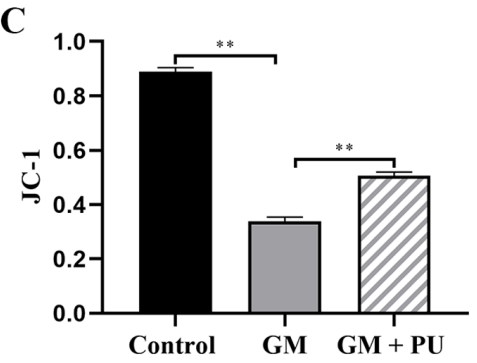

E

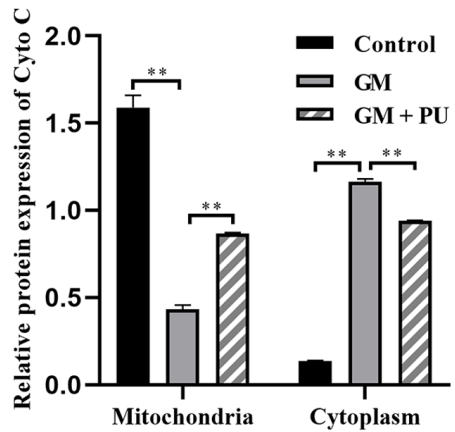

Figure 4. Effect of PU on the GM-induced permeability of the mitochondrial membrane in HEI-OC1 cells. Red fluorescence represents the mitochondrial aggregated form of JC-1, indicating intact mitochondrial membrane potential. Green fluorescence represents the monomeric form of JC-1, indicating dissipation of the $\Delta \Psi \mathrm{m}$. (A) Representative photographs of JC-1 staining in different groups. Scale bar, $200 \mu \mathrm{m}$. (B) Flow cytometry study of the mitochondrial membrane potential. (C) Mitochondrial membrane potential analysis. $n=6$. $^{* *} \mathrm{P}<0.01$. (D) Expression of Cyto $\mathrm{C}$ in the mitochondria and cytoplasm of $\mathrm{HEI}-\mathrm{OC} 1$ cells as determined by western blotting. (E) Statistical analysis of protein expression in each group. $\mathrm{n}=6$. $^{* *} \mathrm{P}<0.01$. PU, puerarin; GM, gentamicin; HEI-OC1, House Ear Institute-Organ of Corti 1; Cyto C, cytochrome $c$.

A
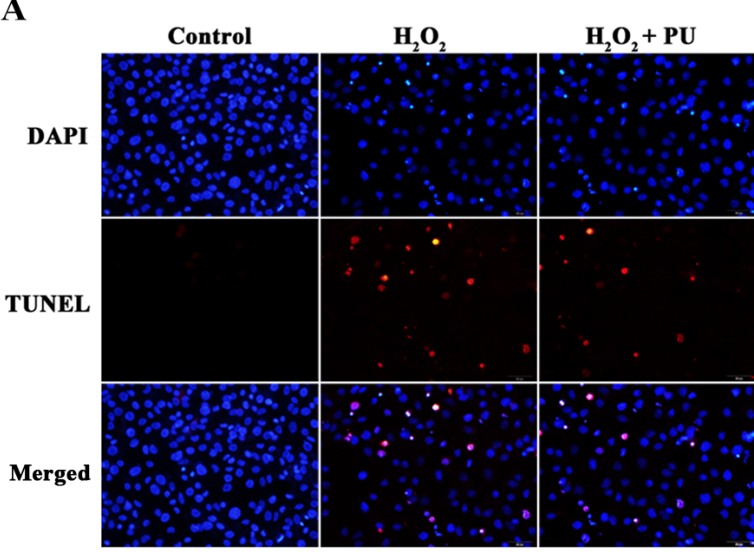
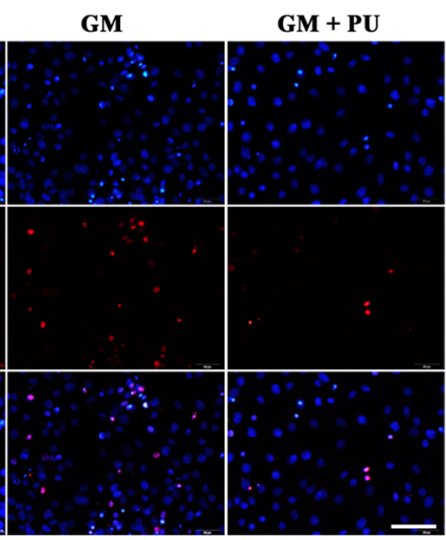

C

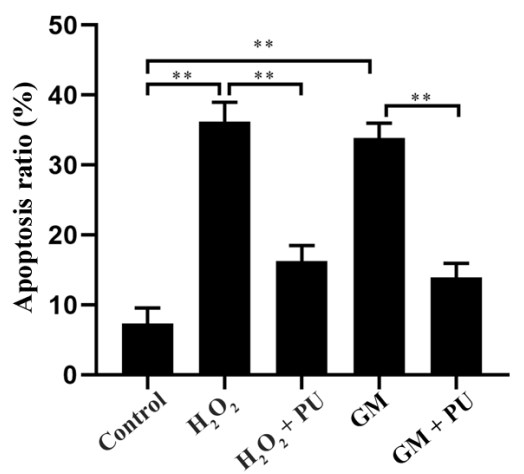

B
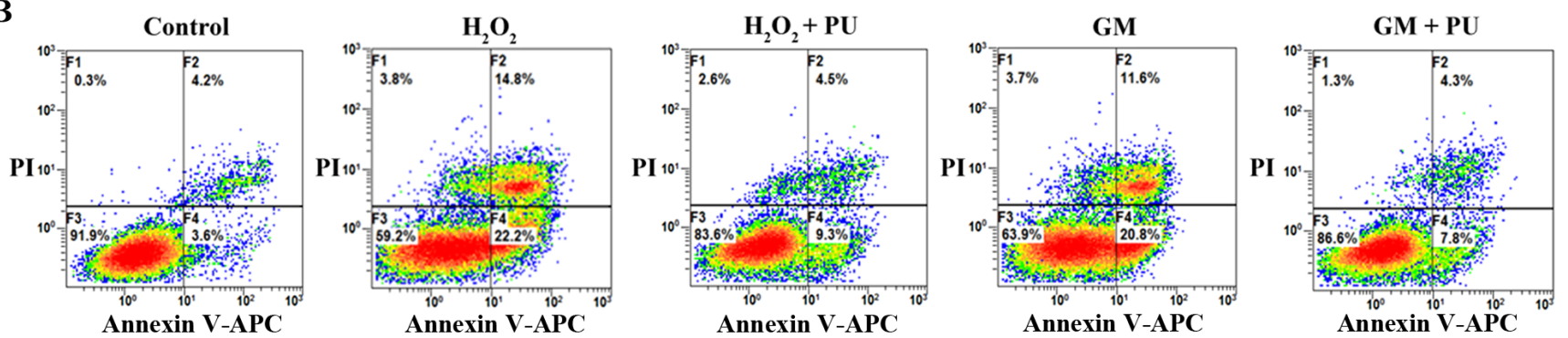

Figure 5. Rate of apoptosis after different treatments as determined by TUNEL assay and flow cytometry. (A) Image of HEI-OC1 cells stained with TUNEL staining. Nuclei were stained with DAPI (blue) and HEI-OC1 cells were stained with TUNEL (red). Scale bar, $100 \mu \mathrm{m}$. (B) Flow cytometry was used to assess apoptosis after treatment under different conditions for 24 h. (C) The apoptotic rate of the cells was statistically analysed. $n=6$. * $P<0.01$. PU, puerarin; GM, gentamicin; HEI-OC1, House Ear Institute-Organ of Corti 1.

the TUNEL method (Fig. 5A) and flow cytometry (Fig. 5B). The results showed that apoptosis was significantly increased after $\mathrm{H}_{2} \mathrm{O}_{2}$ and $\mathrm{GM}$ treatment and that PU pre-treatment significantly reduced this apoptosis (Fig. 5C). The RT-qPCR 
A

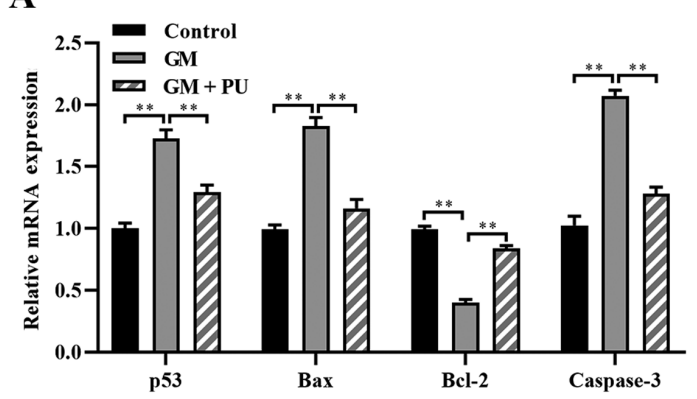

C

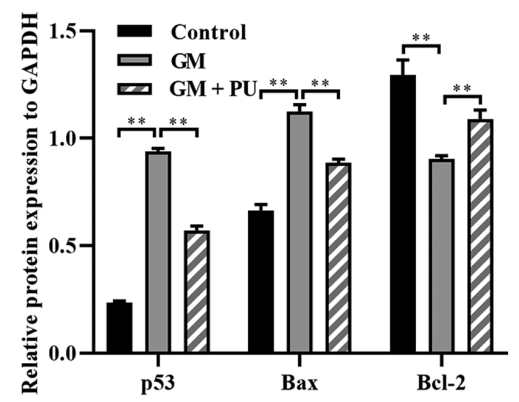

D

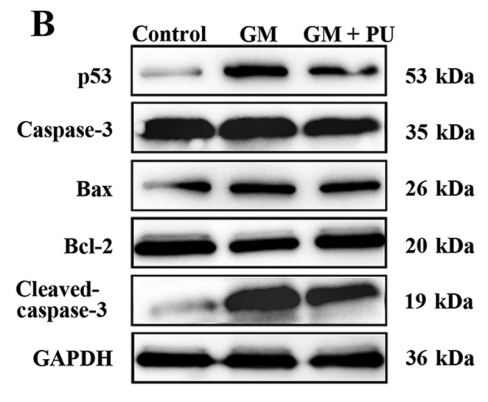

$\mathbf{E}$
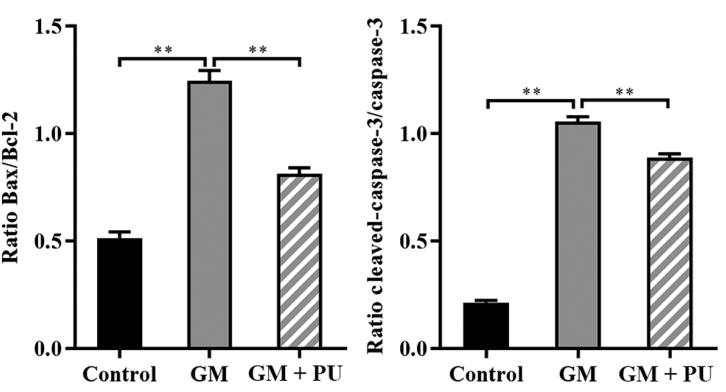

Figure 6. Expression levels of caspase-3, cleaved-caspase-3, Bcl-2, Bax and p53 were analysed via RT-qPCR and western blotting. (A) The mRNA expression levels of caspase-3, Bcl-2, Bax and p53 in each group were detected by RT-qPCR. $\mathrm{n}=6$. ${ }^{* *} \mathrm{P}<0.01$. (B) The relative expression levels of p53, caspase-3, Bax, Bcl-2 and cleaved-caspase-3 were analysed via western blotting. (C) Statistical analysis of protein expression in each group. $\mathrm{n}=6$. ${ }^{* *} \mathrm{P}<0.01$. (D) The Bax to Bcl-2 ratio. Statistical analysis of protein expression $\mathrm{n}=6$. $^{* *} \mathrm{P}<0.01$. (E) The cleaved-caspase-3 to caspase-3 ratio. Statistical analysis of protein expression. $\mathrm{n}=6$. ${ }^{* *} \mathrm{P}<0.01$. PU, puerarin; GM, gentamicin; RT-qPCR, reverse transcription-quantitative PCR.

results suggested that compared with the GM group, the $\mathrm{GM}+\mathrm{PU}$ group exhibited significantly reduced mRNA expression of Bax, caspase-3 and p53, and increased mRNA expression of Bcl-2 (Fig. 6A). The western blotting results (Fig. 6B) showed that p53 expression was increased after the HEI-OC1 cells were treated with GM; moreover, PU could decrease the GM-induced expression of p53. Compared with that in the GM group, the Bax and cleaved-caspase- 3 expression in the GM + PU group was significantly decreased, while the Bcl-2 expression was increased (Fig. 6C). Moreover, the $\mathrm{Bax} / \mathrm{Bcl}-2$ ratio and cleaved-caspase-3/caspase-3 ratio in the GM + PU group was significantly lower than that in the GM group (Fig. 6D and E).

\section{Discussion}

PU is mainly used in the treatment of neurological diseases, cardiovascular diseases and hepatic impairment (7). In the present study, it was found that PU could protect the hearing of GM-treated C57BL/6J mice. We will build more samples and conduct further animal experiments based on this mouse model in follow-up studies. The HEI-OC1 cell line is extremely sensitive to ototoxic drugs. Moreover, the HEI-OC1 cell line is a very useful cell model that has been widely used in a number of studies investigating the molecular mechanism underlying ototoxicity and in studies that screen novel drugs with protective effects in vitro $(27,28)$. After treatment with high concentrations of PU, cell viability was decreased. This finding indicated the potential toxicity of high concentrations of PU. We will conduct further in-depth research on this toxicity in the future.

The main sites of aminoglycoside aggregation in cochlear hair cells are the mitochondria, which are the main organelles that produce oxygen free radicals (29). Appropriate ROS levels can promote immunity, repair and growth of the body, but excessive ROS levels can cause damage to mitochondria and lead to programmed cell death (30). ROS include $\mathrm{H}_{2} \mathrm{O}_{2}, \mathrm{OH}-$, $\mathrm{NO}$ and ONOO-. Among these ROS, $\mathrm{H}_{2} \mathrm{O}_{2}$ easily penetrates the plasma membrane and produces the highly reactive free radical $\mathrm{OH}-$, which leads to damage to cells and tissues and induces cell apoptosis. $\mathrm{H}_{2} \mathrm{O}_{2}$ has been widely used to establish in vitro models of oxidative stress damage (31). In the present study, $\mathrm{H}_{2} \mathrm{O}_{2}$ was used to establish a control group for oxidative stress injury.

Although the mechanism underlying GM-induced injury has not been determined, ROS may be key players in this mechanism (32), and ROS can also cause oxidative stress and cell damage. The ototoxicity induced by GM is related to the production of ROS, which leads to damage to the auditory hair cells in Corti organs. For a long time, cochlear sensory cells have been known to produce ROS after exposure to aminoglycoside compounds (33). p53 is an important protein that mediates cell apoptosis, and p53 is directly regulated by redox signals due to its readily oxidized cysteine (16). Previous studies have shown that p53 is activated when ROS levels increase $(16,34)$. Moreover, p53 expression has been demonstrated to be positively correlated with Bax expression (35). Bax not only antagonizes the inhibitory effect of Bcl-2 on apoptosis, but also promotes apoptosis. Caspase-3 is a regulator of cell death and plays an important role in the progression of apoptosis. Caspase- 3 is a protease that directly leads to the disintegration of apoptotic cells and plays a central role in the network of apoptotic mechanisms. As an upstream protein that regulates caspase-3, Bax can activate caspase- 3 and initiate the caspase cascade (36). The cytotoxicity of GM 
in hair cells is mainly due to the initiation of the mitochondrial apoptosis pathway (29). In the present study, p53 expression was increased, and p53 regulated the expression of Bcl-2 family proteins when ROS accumulated in HEI-OC1 cells. Bax and Bcl-2 form heteropolymers, enhance mitochondrial membrane permeability, release apoptotic factors into the cytoplasm and stimulate the caspase apoptotic pathway, leading to the programmed death of HEI-OC1 cells (11). Investigation into the regulatory roles of ROS and p53 was not performed in the present study, so these results may have limited generalizability. In future studies, p53 and ROS inhibitors will be used to clarify the protective effect of ROS and p53 on PU in auditory hair cell damage induced by GM.

Previous studies have shown that PU has a variety of pharmacological activities and exerts antioxidant effects $(11,37)$. The present study found that PU protected against GM-induced hearing damage in $\mathrm{C} 57 \mathrm{BL} / 6 \mathrm{~J}$ mice and ameliorated the morphological changes in mouse cochlear hair cells after GM-mediated damage. It was also observed that PU could reduce the production of ROS, downregulate the expression of p53, Bax and caspase-3, and upregulate the expression of Bcl-2 in GM- and $\mathrm{H}_{2} \mathrm{O}_{2}$-treated HEI-OC1 cells. After treatment of HEI-OC1 cells with GM and $\mathrm{H}_{2} \mathrm{O}_{2}$, the expression of p53 was significantly increased. It was demonstrated that PU may ameliorate GM-induced ototoxicity, which is at least partially mediated by p53-regulated apoptotic signalling pathways, by inhibiting the mitochondria-dependent apoptosis pathway. This study provided novel insights into potential therapeutic targets for protecting against GM-induced ototoxicity. PU could be used as a protective agent against the ototoxicity caused by GM.

\section{Acknowledgements}

The authors would like to thank Dr Panpan Huang, Dr Wenxiang Fang and Dr Guodong Shen from the University of Science and Technology of China (Hefei, China), for their technical assistance. This work was supported by Anhui Provincial Key Laboratory of Tumor Immunotherapy and Nutrition Therapy (Hefei, China), which provided experimental equipment.

\section{Funding}

This research was supported by the National Natural Sciences Foundation of China (grant nos. 81800911 and 81470699), the Anhui Natural Science Foundation (grant no. 1808085QH248) and Fundamental Research Funds for the Central Universities (grant no. WK9110000053).

\section{Availability of data and materials}

The datasets used and/or analysed during the current study are available from the corresponding author on reasonable request.

\section{Authors' contributions}

PN and YS contributed equally to the work and should be regarded as co-first authors. $\mathrm{CP}$ and Jing $\mathrm{S}$ conceived and designed the study. PN, YS, SW, GL, XT and Jia S performed the cell and animal experiments. PN and YS performed the statistical analysis and drafted the manuscript. CP reviewed and edited the manuscript. PN and CP confirm the authenticity of all the raw data. All the authors read and approved the final manuscript.

\section{Ethics approval and consent to participate}

The animal protocols followed the guidelines of the Institutional Animal Care and Use Committee of University of Science and Technology of China (Jinan, China), and the experiments were conducted in accordance with the National Institutes of Health (NIH) Guide for the Care and Use of Animals in Laboratory Experiments. The study was approved by the Institutional Animal Care and Use Committee of University of Science and Technology of China [approval no. 2020-N(A)-032]. Anhui Provincial Hospital is the other name for the First Affiliated Hospital of the University of Science and Technology of China.

\section{Patient consent for publication}

Not applicable.

\section{Competing interests}

The authors declare that they have no competing interests.

\section{References}

1. Liu J, Kachelmeier A, Dai C, Li H and Steyger PS: Uptake of fluorescent gentamicin by peripheral vestibular cells after systemic administration. PLoS One 10: e0120612, 2015.

2. Ding D, Zhang J, Jiang H, Xuan W, Qi W and Salvi R: Some Ototoxic Drugs Destroy Cochlear Support Cells Before Damaging Sensory Hair Cells. Neurotox Res 37: 743-752, 2020.

3. Yao L, Zhang JW, Chen B, Cai M, Feng D, Wang Q, Wang X, Sun J, Zheng Y, Wang G and Zhou F: Mechanisms and pharmacokinetic/pharmacodynamic profiles underlying the low nephrotoxicity and ototoxicity of etimicin. Acta Pharmacol Sin 41: 866-878, 2020.

4. Kros CJ and Steyger PS: Aminoglycoside- and Cisplatin-Induced Ototoxicity: Mechanisms and Otoprotective Strategies. Cold Spring Harb Perspect Med 9: a033548, 2019.

5. Liu Y, Yu Y, Chu H, Bing D, Wang S, Zhou L, Chen J, Chen Q, Pan C, Sun Y, et al: 17-DMAG induces Hsp70 and protects the auditory hair cells from kanamycin ototoxicity in vitro. Neurosci Lett 588: 72-77, 2015.

6. Yang Q, Zhou Y, Yin H, Li H, Zhou M, Sun G, Cao Z, Man R, Wang $\mathrm{H}$ and Li J: PINK1 Protects Against Gentamicin-Induced Sensory Hair Cell Damage: Possible Relation to Induction of Autophagy and Inhibition of p53 Signal Pathway. Front Mol Neurosci 11: 403, 2018.

7. Mahdy HM, Mohamed MR, Emam MA, Karim AM, Abdel-Naim AB and Khalifa AE: The anti-apoptotic and anti-inflammatory properties of puerarin attenuate 3-nitropropionic-acid induced neurotoxicity in rats. Can J Physiol Pharmacol 92: 252-258, 2014.

8. Umeno A, Horie M, Murotomi K, Nakajima Y and Yoshida Y: Antioxidative and Antidiabetic Effects of Natural Polyphenols and Isoflavones. Molecules 21: 708, 2016.

9. Xiong FL, Sun XH, Gan L, Yang XL and Xu HB: Puerarin protects rat pancreatic islets from damage by hydrogen peroxide. Eur J Pharmacol 529: 1-7, 2006.

10. Kim J, Kim KM, Kim CS, Sohn E, Lee YM, Jo K and Kim JS: Puerarin inhibits the retinal pericyte apoptosis induced by advanced glycation end products in vitro and in vivo by inhibiting NADPH oxidase-related oxidative stress. Free Radic Biol Med 53: 357-365, 2012. 
11. Zhang Y, Yang X, Ge X and Zhang F: Puerarin attenuates neurological deficits via Bcl-2/Bax/cleaved caspase-3 and Sirt3/SOD2 apoptotic pathways in subarachnoid hemorrhage mice. Biomed Pharmacother 109: 726-733, 2019.

12. Liu B, Zhao C, Li H, Chen X, Ding Y and Xu S: Puerarin protects against heart failure induced by pressure overload through mitigation of ferroptosis. Biochem Biophys Res Commun 497: 233-240, 2018

13. Liu S, Cao XL, Liu GQ, Zhou T, Yang XL and Ma BX: The in silico and in vivo evaluation of puerarin against Alzheimer's disease. Food Funct 10: 799-813, 2019.

14. Liu YS, Yuan MH, Zhang CY, Liu HM, Liu JR, Wei AL, Ye Q, Zeng B, Li MF, Guo YP, et al: Puerariae Lobatae radix flavonoids and puerarin alleviate alcoholic liver injury in zebrafish by regulating alcohol and lipid metabolism. Biomed Pharmacother 134: 111121,2021

15. He L, Chen Y, Feng J, Sun W, Li S, Ou M and Tang L: Cellular senescence regulated by SWI/SNF complex subunits through p53/p21 and p16/pRB pathway. Int J Biochem Cell Biol 90: 29-37, 2017.

16. Shi T and Dansen TB: Reactive Oxygen Species Induced p53 Activation: DNA Damage, Redox Signaling, or Both? Antioxid Redox Signal 33: 839-859, 2020.

17. Valverde M, Lozano-Salgado J, Fortini P, Rodriguez-Sastre MA Rojas E and Dogliotti E: Hydrogen Peroxide-Induced DNA Damage and Repair through the Differentiation of Human Adipose-Derived Mesenchymal Stem Cells. Stem Cells Int 2018: 1615497, 2018

18. National Research Council: Guide for the Care and Use of Laboratory Animals. National Academies Press, Washington, DC, 2010. https://www.ncbi.nlm.nih.gov/books/NBK54050/.

19. Chen X, Qian L, Wang B, Zhang Z, Liu H, Zhang Y and Liu J: Synergistic Hypoglycemic Effects of Pumpkin Polysaccharides and Puerarin on Type II Diabetes Mellitus Mice. Molecules 24 $955,2019$.

20. Zhang $\mathrm{X}$ and $\mathrm{Yu}$ J: Baicalin attenuates gentamicin-induced cochlear hair cell ototoxicity. J Appl Toxicol 39: 1208-1214, 2019.

21. Svorc P Jr, Bačová I, Svorc P and Bužga M: Autonomic nervous system under ketamine/ xylazine and pentobarbital anaesthesia in a Wistar rat model: a chronobiological view. Prague Med Rep 114: 72-80, 2013.

22. Eruslanov E and Kusmartsev S: Identification of ROS using oxidized DCFDA and flow-cytometry. Methods Mol Biol 594: $57-72,2010$.

23. Lin H, Xiong H, Su Z, Pang J, Lai L, Zhang H, Jian B, Zhang W and Zheng Y: Inhibition of DRP-1-Dependent Mitophagy Promotes Cochlea Hair Cell Senescence and Exacerbates Age-Related Hearing Loss. Front Cell Neurosci 13: 550, 2019.

24. Yu X, Liu W, Fan Z, Qian F, Zhang D, Han Y, Xu L, Sun G, Qi J, Zhang S, et al: c-Myb knockdown increases the neomycin-induced damage to hair-cell-like HEI-OC1 cells in vitro. Sci Rep 7: 41094, 2017.
25. Livak KJ and Schmittgen TD: Analysis of relative gene expression data using real-time quantitative PCR and the 2(-Delta Delta C(T)) Method. Methods 25: 402-408, 2001.

26. Stevens JM: Cytochrome $c$ as an experimental model protein. Metallomics 3: 319-322, 2011.

27. Kalinec G, Thein P, Park C and Kalinec F: HEI-OC1 cells as a model for investigating drug cytotoxicity. Hear Res 335: 105-117, 2016.

28. Park C, Thein P, Kalinec G and Kalinec F: HEI-OC1 cells as a model for investigating prestin function. Hear Res 335: 9-17, 2016.

29. Kros CJ and Steyger PS: Aminoglycoside- and Cisplatin-Induced Ototoxicity: Mechanisms and Otoprotective Strategies. Cold Spring Harb Perspect Med 9: a033548, 2019.

30. Circu ML and Aw TY: Reactive oxygen species, cellular redox systems, and apoptosis. Free Radic Biol Med 48: 749-762, 2010.

31. Wang Z, Wei D and Xiao H: Methods of cellular senescence induction using oxidative stress. Methods Mol Biol 1048: 135-144, 2013.

32. Choung YH, Taura A, Pak K, Choi SJ, Masuda M and Ryan AF: Generation of highly-reactive oxygen species is closely related to hair cell damage in rat organ of Corti treated with gentamicin. Neuroscience 161: 214-226, 2009.

33. Zhou M, Sun G, Zhang L, Zhang G, Yang Q, Yin H, Li H, Liu W, Bai X, Li J, et al: STK33 alleviates gentamicin-induced ototoxicity in cochlear hair cells and House Ear Institute-Organ of Corti 1 cells. J Cell Mol Med 22: 5286-5299, 2018.

34. Mishina NM, Bogdanova YA, Ermakova YG, Panova AS, Kotova DA, Bilan DS, Steinhorn B, Arnér ESJ, Michel T and Belousov VV: Which Antioxidant System Shapes Intracellular $\mathrm{H}_{2} \mathrm{O}_{2}$ Gradients? Antioxid Redox Signal 31: 664-670, 2019.

35. Qi X, Davis B, Chiang YH, Filichia E, Barnett A, Greig NH, Hoffer B and Luo Y: Dopaminergic neuron-specific deletion of p53 gene is neuroprotective in an experimental Parkinson's disease model. J Neurochem 138: 746-757, 2016.

36. Zhao H, Yenari MA, Cheng D, Sapolsky RM and Steinberg GK: Bcl-2 overexpression protects against neuron loss within the ischemic margin following experimental stroke and inhibits cytochrome $c$ translocation and caspase-3 activity. J Neurochem 85: 1026-1036, 2003

37. Xing ZH, Ma YC, Li XP, Zhang B and Zhang MD: Research progress of puerarin and its derivatives on anti-inflammatory and anti-gout activities. Zhongguo Zhongyao Zazhi 42: 3703-3708, 2017 (In Chinese).

This work is licensed under a Creative Commons Attribution-NonCommercial-NoDerivatives 4.0 International (CC BY-NC-ND 4.0) License. 